\title{
Search for Axionlike Dark Matter Using Solid-State Nuclear Magnetic Resonance
}

Deniz Aybas $\odot,{ }^{1,2}$ Janos Adam, ${ }^{1}$ Emmy Blumenthal, ${ }^{1}$ Alexander V. Gramolin, ${ }^{1}$ Dorian Johnson, ${ }^{1}$ Annalies Kleyheeg, ${ }^{1}$ Samer Afach, ${ }^{3,4}$ John W. Blanchard, ${ }^{3}$ Gary P. Centers, ${ }^{3,4}$ Antoine Garcon, ${ }^{3,4}$ Martin Engler, ${ }^{3,4}$ Nataniel L. Figueroa, ${ }^{3,4}$ Marina Gil Sendra, ${ }^{3,4}$ Arne Wickenbrock, ${ }^{3,4}$ Matthew Lawson, ${ }^{5,6}$ Tao Wang, ${ }^{7}$ Teng Wu, ${ }^{8}$ Haosu Luo, ${ }^{9}$ Hamdi Mani, ${ }^{10}$

Philip Mauskopf ${ }^{10}$ Peter W. Graham, ${ }^{11}$ Surjeet Rajendran, ${ }^{12}$ Derek F. Jackson Kimball, ${ }^{13}$ Dmitry Budker, ${ }^{3,4,14}$ and Alexander O. Sushkov $\oplus^{1,2,15, *}$

${ }^{1}$ Department of Physics, Boston University, Boston, Massachusetts 02215, USA

${ }^{2}$ Department of Electrical and Computer Engineering, Boston University, Boston, Massachusetts 02215, USA

${ }^{3}$ Helmholtz-Institut, GSI Helmholtzzentrum für Schwerionenforschung, 55128 Mainz, Germany

${ }^{4}$ Johannes Gutenberg-Universität Mainz, 55128 Mainz, Germany

${ }^{5}$ The Oskar Klein Centre for Cosmoparticle Physics, Department of Physics, Stockholm University, AlbaNova, 10691 Stockholm, Sweden

${ }^{6}$ Nordita, KTH Royal Institute of Technology and Stockholm University, Roslagstullsbacken 23, 10691 Stockholm, Sweden

${ }^{7}$ Department of Physics, Princeton University, Princeton, New Jersey 08544, USA

${ }^{8}$ State Key Laboratory of Advanced Optical Communication Systems and Networks, Department of Electronics, and Center for Quantum Information Technology, Peking University, Beijing 100871, China

${ }^{9}$ Shanghai Institute of Ceramics, Chinese Academy of Sciences, Shanghai 201800, China

${ }^{10}$ School of Earth and Space Exploration, Arizona State University, Tempe, Arizona 85287, USA

${ }^{11}$ Stanford Institute for Theoretical Physics, Stanford University, Stanford, California 94305, USA

${ }^{12}$ Department of Physics and Astronomy, The Johns Hopkins University, Baltimore, Maryland 21218, USA

${ }^{13}$ Department of Physics, California State University-East Bay, Hayward, California 94542-3084, USA

${ }^{14}$ Department of Physics, University of California, Berkeley, California 94720-7300, USA

${ }^{15}$ Photonics Center, Boston University, Boston, Massachusetts 02215, USA

(Received 29 December 2020; revised 13 January 2021; accepted 9 March 2021; published 9 April 2021)

We report the results of an experimental search for ultralight axionlike dark matter in the mass range 162-166 neV. The detection scheme of our Cosmic Axion Spin Precession Experiment is based on a precision measurement of ${ }^{207} \mathrm{~Pb}$ solid-state nuclear magnetic resonance in a polarized ferroelectric crystal. Axionlike dark matter can exert an oscillating torque on ${ }^{207} \mathrm{~Pb}$ nuclear spins via the electric dipole moment coupling $g_{d}$ or via the gradient coupling $g_{a N N}$. We calibrate the detector and characterize the excitation spectrum and relaxation parameters of the nuclear spin ensemble with pulsed magnetic resonance measurements in a $4.4 \mathrm{~T}$ magnetic field. We sweep the magnetic field near this value and search for axionlike dark matter with Compton frequency within a $1 \mathrm{MHz}$ band centered at $39.65 \mathrm{MHz}$. Our measurements place the upper bounds $\left|g_{d}\right|<9.5 \times 10^{-4} \mathrm{GeV}^{-2}$ and $\left|g_{a N N}\right|<2.8 \times 10^{-1} \mathrm{GeV}^{-1}$ ( $95 \%$ confidence level) in this frequency range. The constraint on $g_{d}$ corresponds to an upper bound of $1.0 \times 10^{-21} e \mathrm{~cm}$ on the amplitude of oscillations of the neutron electric dipole moment and $4.3 \times 10^{-6}$ on the amplitude of oscillations of $C P$-violating $\theta$ parameter of quantum chromodynamics. Our results demonstrate the feasibility of using solid-state nuclear magnetic resonance to search for axionlike dark matter in the neV mass range.

DOI: 10.1103/PhysRevLett.126.141802

The existence of dark matter is indicated by astronomical and cosmological evidence, but its interactions, aside from gravity, remain undetected [1,2]. A number of theoretical models of physics at high energies, such as string theory,

Published by the American Physical Society under the terms of the Creative Commons Attribution 4.0 International license. Further distribution of this work must maintain attribution to the author(s) and the published article's title, journal citation, and DOI. Funded by SCOAP. grand unified theories, and models with extra dimensions, incorporate light pseudoscalar bosons (axionlike particles, ALPs), which are potential dark matter candidates [3-7]. Among these, the axion is particularly compelling, because it also offers a solution to the strong $C P$ problem of quantum chromodynamics (QCD) [7-11]. The axion or axionlike field $a(t)=a_{0} \cos \left(\omega_{a} t\right)$ oscillates at the Compton frequency $\nu_{a}=\omega_{a} /(2 \pi)=m_{a} c^{2} / h$, where $c$ is the speed of light in vacuum, $h$ is the Planck constant, and $m_{a}$ is the unknown ALP mass, which can be in a broad range, roughly between $10^{-21}$ and $10^{-3} \mathrm{eV}$ [12-14]. 
The field amplitude $a_{0}$ is fixed by the assumption that it dominates the dark matter energy density: $\rho_{\text {DM }}=m_{a}^{2} a_{0}^{2} / 2 \approx 3.6 \times 10^{-42} \mathrm{GeV}^{4} \quad[15,16]$. Kinetic energy of the axionlike dark matter field introduces small corrections to its frequency spectrum. The standard halo model predicts the spectral shape with linewidth $\left(v_{0}^{2} / c^{2}\right) \nu_{a} \approx 10^{-6} \nu_{a}$, where $v_{0} \approx 220 \mathrm{~km} / \mathrm{s}$ is the circular rotation speed of the Milky Way Galaxy at the Sun's location $[17,18]$.

Experimental searches for axionlike particles rely on symmetry arguments about the nature of their interactions with standard model particles $[7,16,19,20]$. These interactions are suppressed by a large energy scale, set by the decay constant $f_{a}$, which could lie near the grand unification, or the Planck scale [21]. Most experiments to date have focused on the electromagnetic interaction, which can mix photons with axions and ALPs in the presence of a strong magnetic field [22-32]. The Cosmic Axion Spin Precession Experiments (CASPEr) search for different interactions: the electric dipole moment (EDM) interaction and the gradient interaction with nuclear spin $\boldsymbol{I}$ [19,33-37]. The gradient interaction Hamiltonian is $H_{a N N}=g_{a N N} \nabla a \cdot I$, where $g_{a \mathrm{NN}}$ is the coupling strength. The EDM interaction arises from the defining coupling of the axion to the gluon field [38]. Its Hamiltonian can be written as $H_{\mathrm{EDM}}=g_{d} a \boldsymbol{E}^{*} \cdot \boldsymbol{I} / I$, where $g_{d}$ is the coupling strength and $E^{*}$ is an effective electric field [19]. This interaction is equivalent to that of a parity- and time-reversal-violating oscillating EDM, given by $d=g_{d} a_{0} \cos \left(\omega_{a} t\right)$. This corresponds to an oscillating QCD $\theta$ parameter: $\theta(t)=\left(a_{0} / f_{a}\right) \cos \left(\omega_{a} t\right)$, with $g_{d}$ inversely proportional to $f_{a}[16,39]$. The EDM coupling generates axion mass, and for the QCD axion $m_{a} \approx \Lambda_{\mathrm{QCD}}^{2} / f_{a}$, where $\Lambda_{\mathrm{QCD}} \approx 200 \mathrm{MeV}$ is the QCD confinement scale $[16,40]$.

The sensitivity of static EDM experiments to the oscillating EDM is suppressed, although data reanalysis has produced limits at low frequencies [41,42]. Astrophysical constraints can be derived by analyzing the cooling dynamics of the supernova SN1987A $[16,43]$. Constraints can also be extracted from analysis of ${ }^{4} \mathrm{He}$ production during big bang nucleosynthesis [44] and from analysis of black hole superradiance [45]. CASPErelectric is a direct, model-independent search for the EDM and gradient interactions of axionlike dark matter, with the potential to reach the sensitivity to the QCD axion [19]. We search for the effects of these interactions on the dynamics of a spin ensemble in a solid with broken inversion symmetry [46-52]. The measurements focus on ${ }^{207} \mathrm{~Pb}^{2+}$ ions, with nuclear spin $I=1 / 2$, in a poled ferroelectric PMN-PT crystal with the chemical formula $\left(\mathrm{PbMg}_{1 / 3} \mathrm{Nb}_{2 / 3} \mathrm{O}_{3}\right)_{2 / 3}-\left(\mathrm{PbTiO}_{3}\right)_{1 / 3}$ (see Supplemental Material [53]). The noncentrosymmetric position of the ions in this crystal gives rise to a large effective electric field, analogous to the effect in polar molecules [73-75].
The EDM or gradient interaction with axionlike dark matter creates an oscillating torque on the nuclear spins. We quantify the magnitude of this torque by the Rabi frequency $\Omega_{a}$, which is proportional to the corresponding interaction strength. For a spin ensemble polarized by an external bias magnetic field, this torque tilts the spins, if it is resonant with their Larmor frequency. The experimental observable is the oscillating transverse magnetization

$$
M_{a}=u M_{0} \Omega_{a} T_{2} \cos \left(\omega_{a} t\right),
$$

where $M_{0}$ is the equilibrium magnetization of the ${ }^{207} \mathrm{~Pb}$ nuclear spin ensemble, $T_{2}$ is the nuclear spin coherence time, and $u$ is a dimensionless spectral factor that takes into account the inhomogeneous broadening of the spin ensemble and the detuning between the ALP Compton frequency and the spin Larmor frequency [53].

Our apparatus makes use of inductive detection to measure the ${ }^{207} \mathrm{~Pb}$ spin precession, Fig. 1(a). We poled the cylindrical PMN-PT crystal along its axis, aligned with the $[1,1,1]$ crystal direction. This created the axial effective electric field $\boldsymbol{E}^{*}$, proportional to the remanent polarization $P_{r}$. We mounted the crystal inside a fiberglass tube, so that $\boldsymbol{E}^{*}$ was perpendicular to the vertical bias magnetic field $\boldsymbol{B}_{0}$, created with a superconducting solenoid. A pickup coil, wound around the tube, was coupled to a low-noise cryogenic preamplifier with a tuned matching circuit, Fig. 1(b). We tuned the pickup probe to have its resonance at $39.7 \mathrm{MHz}$ with quality factor 26, and matched its impedance to the $50 \Omega$ input impedance of the preamplifier [53]. A cylindrical copper shield attenuated external sources of radio frequency interference. We performed all experiments with the apparatus submerged in a liquid helium bath at $4.2 \mathrm{~K}$ temperature [53].

We calibrated the pickup probe using ${ }^{207} \mathrm{~Pb}$ pulsed nuclear magnetic resonance (NMR) measurements, Fig. 1(c). The spins were excited by resonant magnetic field pulses, created by delivering current to the $2 \times 3$-turn Helmholtz excitation coil, coupled to a matching circuit, tuned at $42 \mathrm{MHz}$ with a quality factor 2 . The axis of this coil was orthogonal to the pickup coil axis, Fig. 1(a). After each pulse, nuclear spin free induction decay (FID) was measured with the pickup probe, characterized by transfer coefficient $\alpha=V_{1} /\left(\mu_{0} M_{1}\right)$, where $V_{1}$ is the recorded voltage referred to the amplifier input, $M_{1}$ is the transverse sample magnetization, and $\mu_{0}$ is the permeability of free space. Despite our efforts to minimize the inductive and capacitive couplings between the excitation and the pickup coils, we found that the cryogenic preamplifier saturated during excitation pulses, and its recovery time was too long to observe the fast FID [53]. To address this problem, we placed a single-turn cancellation coil near the pickup coil, Fig. 1(a), and delivered to it a compensating current during the excitation pulses. The amplitude and phase of this compensating current were chosen to cancel the current in 

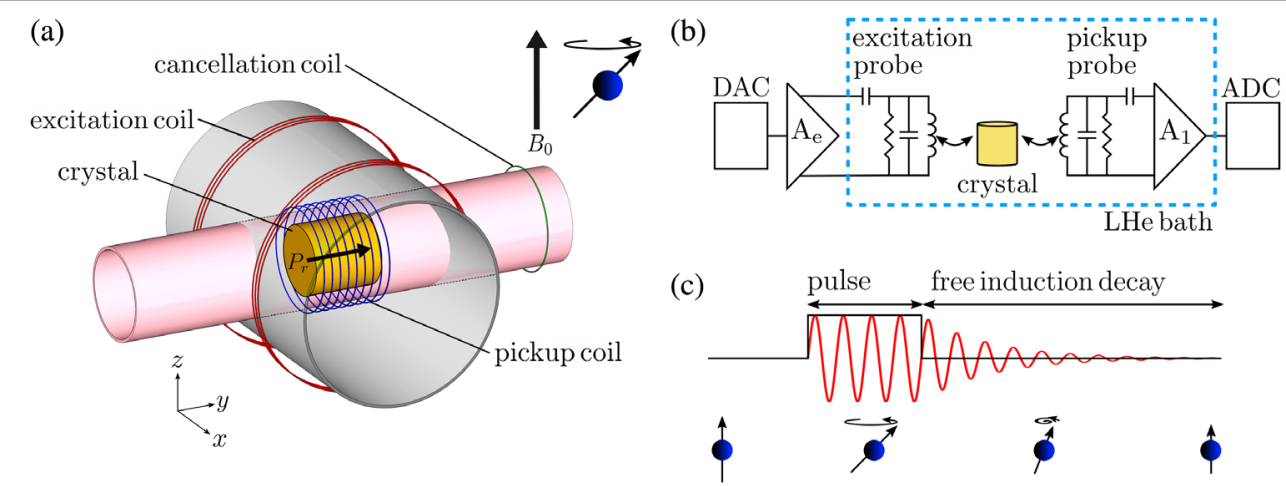

FIG. 1. Experimental setup. (a) The sample was a cylindrical ferroelectric PMN-PT crystal with diameter $0.46 \mathrm{~cm}$ and thickness $0.50 \mathrm{~cm}$. It was electrically polarized along the cylinder axis, indicated with the black arrow. The pickup coil and the cancellation coil were coaxial with the crystal, and the axis of the Helmholtz excitation coil was orthogonal. The vertical leading magnetic field $B_{0}$ set the direction of the equilibrium spin polarization. Coils were supported by G-10 fiberglass cylinders shown in gray and pink. (b) Electrical schematic, showing the excitation and pickup circuits. Excitation pulses generated with the digital-to-analog converter (DAC) were amplified $\left(A_{e}\right)$ and coupled to the excitation coil via a tuned tank circuit that included matching and tuning capacitors, as well as a resistor to set the circuit quality factor. The pickup probe was also designed as a tuned tank circuit, coupling the voltage induced in the pickup coil to a low-noise cryogenic amplifier $\left(A_{1}\right)$, whose output was filtered, further amplified, and digitized with an analog-to-digital converter (ADC). (c) Pulsed NMR sequence used for FID measurements. The spin-ensemble equilibrium magnetization, initially parallel to $B_{0}$, was tilted into the transverse plane by the excitation pulse. The FID signal was recorded after the excitation pulse, as the magnetization precessed and its transverse component decayed.

the pickup probe during excitation and prevent preamplifier saturation, without affecting spin excitation. This scheme is a substitute for the transmit-receive switch, often used in NMR detectors.

We performed the NMR calibration measurements at the leading magnetic field $B_{0}=4.4 \mathrm{~T}$, for which the value of the equilibrium thermal magnetization $M_{0}$ of the spin ensemble was $\mu_{0} M_{0}=2.9 \mathrm{nT}$ [53]. We set the excitation carrier frequency to $39.71 \mathrm{MHz}$ and recorded the FID signals after excitation pulses of variable width. The Fourier spectrum of one of these FID signals is shown in Fig. 2(a). We modeled the FID line shapes by numerically solving the Bloch equations for a spin ensemble with an inhomogeneously broadened excitation spectrum [53]. By fitting the data, we extracted the transverse coherence time of the nuclear spins: $T_{2}=(16.7 \pm 0.9) \mathrm{ms}$, and the pickupcircuit transfer coefficient $\alpha=(2.3 \pm 0.2) \times 10^{4} \mathrm{~V} / \mathrm{T}$. We note that the overall FID spectral width is much greater than $1 / T_{2}$, since the tilting pulse excites a broad frequency band within the inhomogeneous spin distribution. The exact shape of the FID Fourier spectrum depends on the interplay between the excitation-pulse spectrum, the distribution of tipping angles across the spin ensemble, and the $T_{2}$ coherence time.

We measured the inhomogeneous broadening of the ${ }^{207} \mathrm{~Pb}$ nuclear spins in the sample by sweeping the excitation-pulse carrier frequency and recording the corresponding FID spectra. The resulting NMR excitation spectrum was centered at $39.71 \mathrm{MHz}$ and had a full width $\Gamma /(2 \pi)=(78 \pm 2) \mathrm{kHz}$, Fig. 2(b). This broadening is consistent with the chemical shift anisotropy (CSA) of
${ }^{207} \mathrm{~Pb}$ observed in solid-state NMR [76]. We measured the population relaxation time $T_{1}$ of the ${ }^{207} \mathrm{~Pb}$ nuclear spin ensemble with a saturation-recovery measurement, obtaining $T_{1}=(25.8 \pm 0.6) \min [53]$.

The spin evolution in our pulsed NMR calibration measurements was more complicated than the continuous wave-like small spin-tip angle response to axionlike dark matter, described by Eq. (1). In order to confirm the validity of our NMR model in the limit of small spin-tip angles, we recorded and analyzed FID data for a range of excitation Rabi frequencies $\Omega_{e}$. For these measurements, we kept the excitation-pulse width at $20 \mathrm{~ms}$-approximately the coherence time of axionlike dark matter field with Compton frequency near $40 \mathrm{MHz}$. At small excitation amplitudes, the spin response was linear in $\Omega_{e}$, as described by Eq. (1) for the case of the drive due to interaction with axionlike dark matter, Fig. 2(c). The slope of the linear response is proportional to the spectral factor $u=(3.8 \pm 0.3) \times 10^{-4}$, which is well approximated by the ratio of the homogeneous linewidth $\pi / T_{2}$ and the inhomogeneously broadened excitation spectrum width $\Gamma$ [53]. The deviation from linearity at larger $\Omega_{e}$ is due to saturation of the resonant spins in the excitation spectrum, consistent with our Bloch-equation simulations.

Prior to any measurements, the PMN-PT crystal was ferroelectrically poled at room temperature by applying $3.5 \mathrm{kV}$ across the crystal faces. We measured the ferroelectric hysteresis loop by sweeping the applied voltage while recording the current flowing through the sample and integrating it to find the polarization, Fig. 2(d). The resulting value of remanent polarization was $P_{r}=(22 \pm 2) \mu \mathrm{C} / \mathrm{cm}^{2}$. 


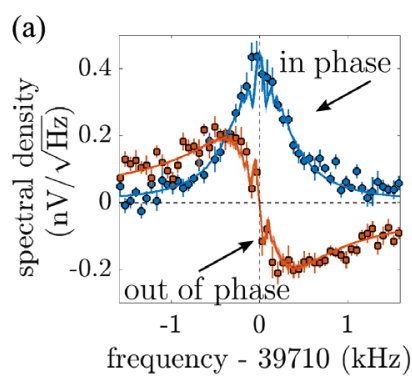

(c)

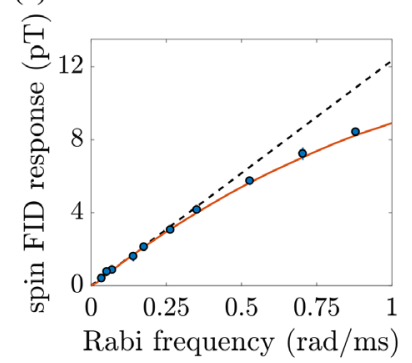

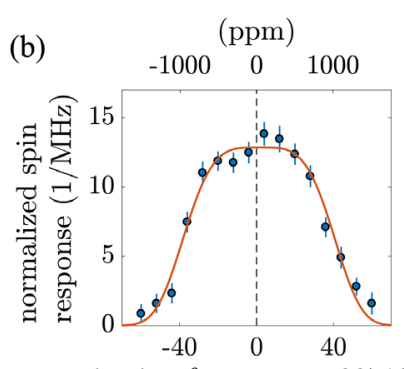

excitation frequency - 39710

$(\mathrm{kHz})$

(d)

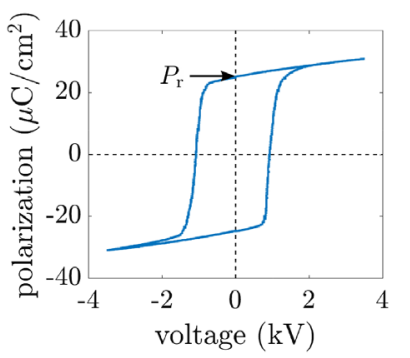

FIG. 2. Sensitivity calibration. (a) Measurements of ${ }^{207} \mathrm{~Pb}$ FID following a spin excitation pulse of length $t_{p}=20 \mathrm{~ms}$. The excitation carrier frequency was set to $39.71 \mathrm{MHz}$, and the Rabi frequency was $\Omega_{e}=0.88 \mathrm{rad} / \mathrm{ms}$. The data points show the inphase (blue circles) and the out-of-phase (orange squares) quadratures of the Fourier transform of the detected voltage, referred to the input of the pickup probe amplifier $A_{1}$. Data points were binned and averaged, the error bars show one standard deviation for each bin. The lines show the best-fit simulation of the spin response, with the light-colored narrow bands indicating the range of simulation results if parameters are varied by one standard deviation away from their best-fit values. We performed the fitting simultaneously to three FID datasets, with excitation-pulse lengths $t_{p}=0.2,2,20 \mathrm{~ms}$, with free parameters including the spin coherence time $T_{2}$ and pickupcircuit transfer coefficient $\alpha$ (see Supplemental Material [53]). (b) Measurement of the normalized ${ }^{207} \mathrm{~Pb}$ NMR excitation spectrum near Larmor frequency 39.71 MHz. Excitation pulses of length $1.6 \mathrm{~ms}$ and Rabi frequency $\Omega_{e}=0.88 \mathrm{rad} / \mathrm{ms}$ were delivered at the carrier frequencies shown on the $x$ axis. Data points show the amplitude of the spin FID response, normalized so that the integral of the spectrum is unity. The error bars indicate one standard deviation uncertainties of the FID spectrum fits. We model the excitation spectrum as a super-Gaussian of order 2 (red line) [53]. (c) Detector calibration for varying drive Rabi frequency. Data points show the amplitude of the spin FID response after an excitation pulse of length $20 \mathrm{~ms}$, delivered at the carrier frequency $39.71 \mathrm{MHz}$, with Rabi frequency $\Omega_{e}$ plotted on the $x$ axis. The error bars indicate one standard deviation uncertainties, obtained by grouping 100 consecutive FID measurements taken at each $\Omega_{e}$ into five sets and independently analyzing each set [53]. The orange line shows the spin response simulated using the Bloch equations with parameters extracted from data in (a). (d) Measurement of ferroelectric hysteresis in the PMN-PT single crystal. The remanent polarization $P_{r}$ persists after the applied voltage has been ramped down to zero.

We recorded hysteresis data before and after the experiments searching for axionlike dark matter, and verified that the fractional degradation of polarization due to thermal cycling and fatigue was smaller than the quoted uncertainty. The effective electric field $E^{*}$ is proportional to the ferroelectric polarization $[48,73,74]$. In order to calculate the value of $E^{*}$ we considered the Schiff moment $S$ of the ${ }^{207} \mathrm{~Pb}$ nucleus, induced by the oscillating QCD $\theta$ parameter $[77,78]$. The dominant contribution to the Schiff moment arises from the parity- and time-reversal-violating nuclear forces, resulting in the value $S=0.04 \theta e \mathrm{fm}^{3}$ [53,79-83]. This corresponds to the magnitude of effective electric field $E^{*}=340 \mathrm{kV} / \mathrm{cm}$. We estimate the theoretical uncertainty in $E^{*}$ on the level of $50 \%$ (see Supplemental Material [53]).

In order to search for axionlike dark matter, we swept the leading magnetic field $B_{0}$ in 21 steps, corresponding to the search frequency range 39.1-40.2 $\mathrm{MHz}$. The step size was chosen to correspond to $50 \mathrm{kHz}$, on the order of the width of the ${ }^{207} \mathrm{~Pb}$ nuclear spin excitation spectrum, Fig. 2(b). The broad NMR excitation spectrum reduced the necessary number of magnetic field steps for a given search frequency range. At each value of $B_{0}$, we recorded $58 \mathrm{~s}$ of scan data sensitive to axionlike dark matter, followed by $58 \mathrm{~s}$ of rescan data that were used in our analysis to identify statistical fluctuations. In order to confirm the experimental calibration, we performed pulsed NMR measurements at three values of the leading field, corresponding to the extremes and the midpoint of the search frequency range [53].

Data analysis consisted of several processing, correction, and signal-search steps. At each value of the leading field $B_{0}$, we divided the recorded scan data into 27 blocks, each of $2.15 \mathrm{~s}$ duration, chosen to be much longer than the $\approx 25 \mathrm{~ms}$ coherence time of any potential ALP dark matter signal in our frequency range. We used the pickup-circuit transfer coefficient $\alpha$ to convert the recorded voltage values to magnetization and performed a discrete Fourier transform on each block, subsequently averaging the power spectral densities (PSDs) of the blocks. Many of the spectra were contaminated with narrow band radio frequency interference that penetrated our electromagnetic shielding. We used Savitzky-Golay digital filtering to identify and reject these narrow band features, while preserving potential axionlike dark matter signals, whose spectral shape is predicted by the standard halo model $[25,53,84]$.

We then processed the data to search for signals due to the EDM and the gradient interactions. The first step was optimal filtering, performed by convolving the PSD with the signal line shape predicted for the corresponding interaction [53]. At each value of $B_{0}$, we retained the optimally filtered data points in a frequency bin, centered at the corresponding Larmor frequency, with full width $80 \mathrm{kHz}$, covering the excitation spectrum bandwidth. We modeled the histogram of these data points as the normal 
(a)

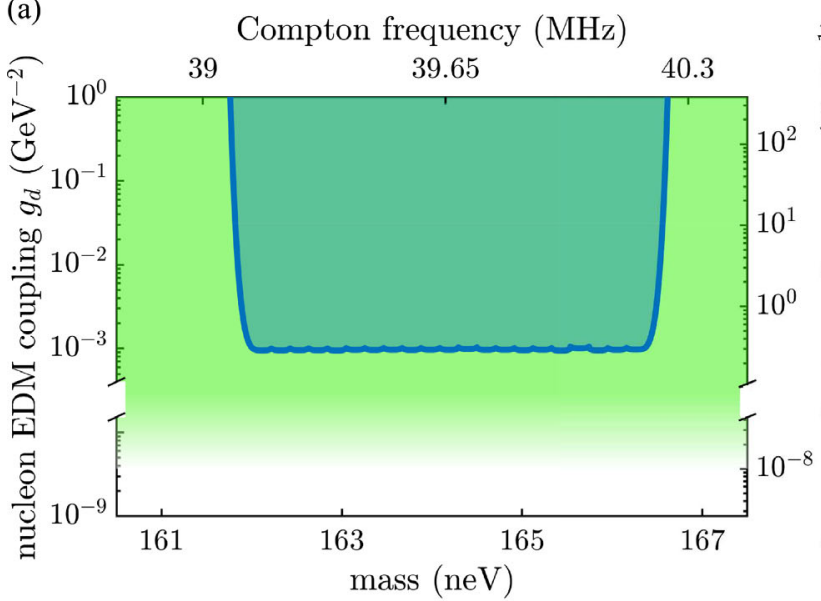

(b)

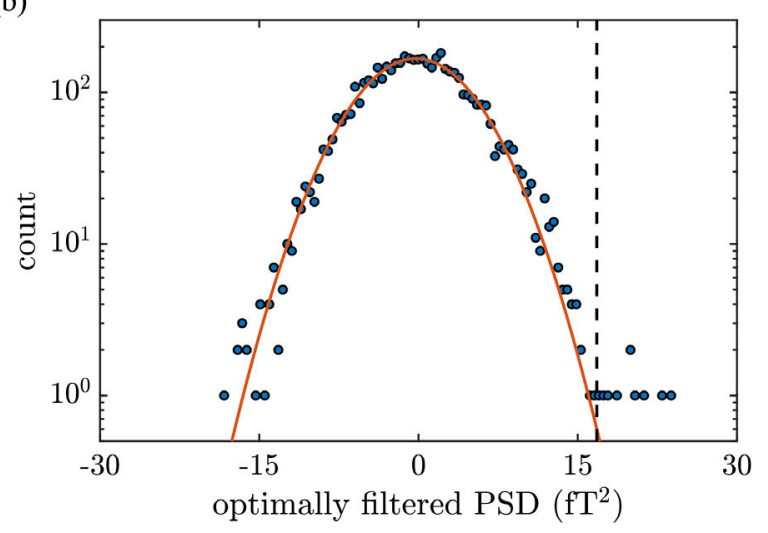

FIG. 3. Results of the search for spin interactions with axionlike dark matter. (a) The axionlike dark matter EDM coupling (left $y$ axis) and nucleon gradient coupling (right $y$ axis) limits in the mass range 162-166 neV shown with a blue line. The shaded region above the line is excluded at $95 \%$ confidence level. The green region is excluded by analysis of cooling of the supernova SN1987A; the color gradient indicates theoretical uncertainty [16]. Existing bounds at other masses, as well as CASPEr sensitivity projections, are shown in Fig. S9 of the Supplemental Material [53]. (b) The histogram of the optimally filtered power spectral density of transverse sample magnetization within the frequency window centered at $39.16 \mathrm{MHz}$. The red line shows the Gaussian distribution model, and the vertical black dashed line shows the $3.355 \sigma$ candidate threshold at $17 \mathrm{fT}^{2}$.

distribution with standard deviation $\sigma$, Fig. 3(b). We set the candidate detection threshold to $3.355 \sigma$, equivalent to $95 \%$ confidence interval for a $5 \sigma$ detection, and flagged all points above the threshold as candidates $[32,53,84]$.

There were 617 candidates for EDM coupling (636 for gradient coupling). In order to reject residual radio frequency interference, we used the fact that radio frequency pickup is independent of the leading field $B_{0}$, while an axionlike dark matter signal should only appear when $B_{0}$ is tuned to a value such that the spin excitation spectrum overlaps with the ALP Compton frequency. We compared the candidates from datasets taken at different values of $B_{0}$, rejecting 569 candidates for EDM coupling (577 for gradient coupling). The remaining 48 candidates for EDM coupling (59 for gradient coupling) were shown to be statistical fluctuations, using a scan-rescan analysis [53]. The search sensitivity was limited by the $\approx 0.05 \mathrm{nV} / \sqrt{\mathrm{Hz}}$ input noise level of the amplifier, corresponding to a magnetic field sensitivity of $\approx 2 \mathrm{fT} / \sqrt{\mathrm{Hz}}$.

Our search did not yield a discovery of the EDM coupling $g_{d}$ or the gradient coupling $g_{a N N}$ of axionlike dark matter. In the absence of a detection, in each frequency bin the $95 \%$ confidence interval limit on magnitudes of these coupling constants corresponds to the $5 \sigma$ value in the Gaussian distribution of the optimally filtered PSD $[32,53,84]$. The limits were corrected to take into account spin saturation [85], normalized by the NMR excitation spectrum for each bin, and concatenated to produce constraints on $g_{d}$ and $g_{a N N}$ over the entire frequency search range, Fig. 3(a). Over the frequency range $39.1-40.2 \mathrm{MHz}$, the constraint on $\left|g_{d}\right|$ is $\left|g_{d}\right|<9.5 \times 10^{-4} \mathrm{GeV}^{-2}$, corresponding to an upper bound of $1.0 \times 10^{-21} e \mathrm{~cm}$ on the amplitude of oscillations of the neutron electric dipole moment and $4.3 \times 10^{-6}$ on the amplitude of oscillations of the QCD $\theta$ parameter. The constraint on $\left|g_{a N N}\right|$ is $\left|g_{a N N}\right|<2.8 \times 10^{-1} \mathrm{GeV}^{-1}$. The uncertainty on these limits is dominated by the theoretical uncertainty in the effective electric field. We are not aware of any existing experimental limits on these interactions in this ALP mass range. Analysis of cooling dynamics of supernova SN1987A can be used to estimate bounds $g_{d} \lesssim 10^{-8} \mathrm{GeV}^{-2}$ and $g_{a N N} \lesssim 10^{-9} \mathrm{GeV}^{-1} \quad[19,24,43]$. However, these model-dependent bounds are subject to significant caveats and uncertainties and may be evaded altogether, reinforcing the importance of laboratory searches [86,87]. Stringent experimental limits on $g_{d}$ and $g_{a N N}$ exist at much lower ALP masses $[35,36,41,42,88-91]$.

There are several ways to improve experimental sensitivity to axionlike dark matter. Since the CSA-induced inhomogeneous broadening is proportional to the Larmor frequency, searching in a lower ALP mass range will reduce the linewidth and therefore improve the search sensitivity. A search in the lower mass range will likely also benefit from superconducting detectors, such as superconducting quantum interference devices and quantum up-converters [92]. Manipulation of light-induced transient paramagnetic centers may enable control over the nuclear spin population-relaxation time $T_{1}$ and nuclear spin hyperpolarization using dynamic polarization techniques. A dramatic sensitivity improvement could be achieved by scaling up the sample volume. We estimate that, with a sample size of $\approx 80 \mathrm{~cm}$, it may be possible to reach the sensitivity necessary to detect the QCD axion $g_{d}$ coupling strength in the mass range between $\approx \mathrm{peV}$ and $\approx 5 \mathrm{neV}$. 
The authors thank Oyku Acican for her help with Fig. 1(a), and Alexander Wilzewski, Hendrik Bekker, O. P. Sushkov, and V. Flambaum for valuable contributions and discussions. The authors acknowledge support from U.S. Department of Energy Award No. DESC0019450, the Heising-Simons Foundation Grant No. 2015-039, the Simons Foundation Grant No. 641332, and the Alfred P. Sloan Foundation Grant No. FG-2016-6728. The work of the Mainz group was supported by the Cluster of Excellence PRISMA+ funded by the German Research Foundation (DFG) within the German Excellence Strategy (Project ID 39083149), by the European Research Council (ERC) under the European Union Horizon 2020 Research and Innovation Program (project Dark-OST, Grant Agreement No. 695405), and by the DFG Reinhart Koselleck project. D. F. J. K. acknowledges the support of the National Science Foundation under Grant No. PHY-1707875.

*asu@bu.edu

[1] D. N. Spergel, Science 347, 1100 (2015).

[2] G. Bertone and T. M. P. Tait, Nature (London) 562, 51 (2018).

[3] J. Preskill, M. B. Wise, and F. Wilczek, Phys. Lett. 120B, 127 (1983).

[4] L. Abbott and P. Sikivie, Phys. Lett. 120B, 133 (1983).

[5] M. Dine and W. Fischler, Phys. Lett. 120B, 137 (1983).

[6] P. Svrcek and E. Witten, J. High Energy Phys. 06 (2006) 051.

[7] I. G. Irastorza and J. Redondo, Prog. Part. Nucl. Phys. 102, 89 (2018).

[8] R. D. Peccei and H. R. Quinn, Phys. Rev. Lett. 38, 1440 (1977).

[9] S. Weinberg, Phys. Rev. Lett. 40, 223 (1978).

[10] F. Wilczek, Phys. Rev. Lett. 40, 279 (1978).

[11] D. DeMille, J. M. Doyle, and A. O. Sushkov, Science 357, 990 (2017).

[12] P. W. Graham and A. Scherlis, Phys. Rev. D 98, 035017 (2018).

[13] A. Ernst, A. Ringwald, and C. Tamarit, J. High Energy Phys. 02 (2018) 103.

[14] K. Schutz, Phys. Rev. D 101, 123026 (2020).

[15] M. Tanabashi et al. (Particle Data Group), Phys. Rev. D 98, 030001 (2018).

[16] P. W. Graham and S. Rajendran, Phys. Rev. D 88, 035023 (2013).

[17] M. S. Turner, Phys. Rev. D 42, 3572 (1990).

[18] N. W. Evans, C. A. J. O'Hare, and C. McCabe, Phys. Rev. D 99, 023012 (2019).

[19] D. Budker, P. W. Graham, M. Ledbetter, S. Rajendran, and A. O. Sushkov, Phys. Rev. X 4, 021030 (2014).

[20] A. Arvanitaki and A. A. Geraci, Phys. Rev. Lett. 113, 161801 (2014).

[21] P. W. Graham and A. Scherlis, Phys. Rev. D 98, 035017 (2018).

[22] P. Sikivie, Phys. Rev. Lett. 51, 1415 (1983).

[23] N. Du et al., Phys. Rev. Lett. 120, 151301 (2018).
[24] P. W. Graham, I. G. Irastorza, S. K. Lamoreaux, A. Lindner, and K. A. van Bibber, Annu. Rev. Nucl. Part. Sci. 65, 485 (2015).

[25] B. M. Brubaker et al., Phys. Rev. Lett. 118, 061302 (2017).

[26] J. Choi, H. Themann, M. J. Lee, B. R. Ko, and Y. K. Semertzidis, Phys. Rev. D 96, 061102(R) (2017).

[27] P. Sikivie, N. Sullivan, and D. B. Tanner, Phys. Rev. Lett. 112, 131301 (2014).

[28] S. Chaudhuri, P. W. Graham, K. Irwin, J. Mardon, S. Rajendran, and Y. Zhao, Phys. Rev. D 92, 075012 (2015).

[29] Y. Kahn, B. R. Safdi, and J. Thaler, Phys. Rev. Lett. 117, 141801 (2016).

[30] S. Chaudhuri, K. Irwin, P. W. Graham, and J. Mardon, arXiv:1803.01627.

[31] J. L. Ouellet, C. P. Salemi, J. W. Foster, R. Henning, Z. Bogorad, J. M. Conrad, J. A. Formaggio, Y. Kahn, J. Minervini, A. Radovinsky, N. L. Rodd, B. R. Safdi, J. Thaler, D. Winklehner, and L. Winslow, Phys. Rev. Lett. 122, 121802 (2019).

[32] A. V. Gramolin, D. Aybas, D. Johnson, J. Adam, and A. O. Sushkov, Nat. Phys. 17, 79 (2021).

[33] A. Garcon, D. Aybas, J. W. Blanchard, G. Centers, N. L. Figueroa, P. W. Graham, D. F. J. Kimball, S. Rajendran, M. G. Sendra, A. O. Sushkov, L. Trahms, T. Wang, A. Wickenbrock, T. Wu, and D. Budker, Quantum Sci. Technol. 3, 014008 (2018).

[34] T. Wang, D. F. J. Kimball, A. O. Sushkov, D. Aybas, J. W. Blanchard, G. Centers, S. R. O. Kelley, A. Wickenbrock, J. Fang, and D. Budker, Phys. Dark Universe 19, 27 (2018).

[35] T. Wu, J. W. Blanchard, G. P. Centers, N. L. Figueroa, A. Garcon, P. W. Graham, Derek F. Jackson Kimball, S. Rajendran, Y. V. Stadnik, A. O. Sushkov, A. Wickenbrock, and D. Budker, Phys. Rev. Lett. 122, 191302 (2019).

[36] A. Garcon, J. W. Blanchard, G. P. Centers, N. L. Figueroa, P. W. Graham, D. F. J. Kimball, S. Rajendran, A. O. Sushkov, Y. V. Stadnik, A. Wickenbrock, T. Wu, and D. Budker, Sci. Adv. 5, eaax4539 (2019).

[37] D. F. Jackson Kimball, S. Afach, D. Aybas, J. W. Blanchard, D. Budker, G. Centers, M. Engler, N. L. Figueroa, A. Garcon, P. W. Graham, H. Luo, S. Rajendran, M. G. Sendra, A. O. Sushkov, T. Wang, A. Wickenbrock, A. Wilzewski, and T. Wu, Springer Proc. Phys. 245, 105 (2020).

[38] P. W. Graham and S. Rajendran, Phys. Rev. D 84, 055013 (2011).

[39] M. Pospelov and A. Ritz, Nucl. Phys. B573, 177 (2000).

[40] M. Baldicchi, A. V. Nesterenko, G. M. Prosperi, D. V. Shirkov, and C. Simolo, Phys. Rev. Lett. 99, 242001 (2007).

[41] C. Abel et al., Phys. Rev. X 7, 041034 (2017).

[42] T. S. Roussy, D. A. Palken, W. B. Cairncross, B. M. Brubaker, D. N. Gresh, M. Grau, K. C. Cossel, K. B. Ng, Y. Shagam, Y. Zhou, V. V. Flambaum, K. W. Lehnert, J. Ye, and E. A. Cornell, arXiv:2006.15787.

[43] G. G. Raffelt, in Axions: Theory, Cosmology, and Experimental Searches (Springer, Berlin, Heidelberg, 2008), pp. $51-71$.

[44] K. Blum, R. T. D’Agnolo, M. Lisanti, and B. R. Safdi, Phys. Lett. B 737, 30 (2014).

[45] A. Arvanitaki, S. Dimopoulos, S. Dubovsky, N. Kaloper, and J. March-Russell, Phys. Rev. D 81, 123530 (2010).

[46] A. J. Leggett, Phys. Rev. Lett. 41, 586 (1978). 
[47] W. Bialek, J. Moody, and F. Wilczek, Phys. Rev. Lett. 56, 1623 (1986).

[48] T. N. Mukhamedjanov and O. P. Sushkov, Phys. Rev. A 72 , 034501 (2005).

[49] D. Budker, S. K. Lamoreaux, A. O. Sushkov, and O. P. Sushkov, Phys. Rev. A 73, 022107 (2006).

[50] A. O. Sushkov, S. Eckel, and S. K. Lamoreaux, Phys. Rev. A 81, 022104 (2010).

[51] K. Z. Rushchanskii, S. Kamba, V. Goian, P. Vanek, M. Savinov, J. Prokleska, D. Nuzhnyy, K. Knízek, F. Laufek, S. Eckel, S. K. Lamoreaux, A. O. Sushkov, M. Lezaić, and N. A. Spaldin, Nat. Mater. 9, 649 (2010).

[52] S. Eckel, A. O. Sushkov, and S. K. Lamoreaux, Phys. Rev. Lett. 109, 193003 (2012).

[53] See Supplemental Material at http://link.aps.org/supplemental/ 10.1103/PhysRevLett.126.141802 for detailed description of the experimental setup, nuclear spin dynamics, and data analysis, which includes Refs. [54-72].

[54] J. B. Miller, B. H. Suits, A. N. Garroway, and M. A. Hepp, Concepts Magn. Reson. 12, 125 (2000).

[55] J. E. Sansonetti and W. C. Martin, J. Phys. Chem. Ref. Data 34, 1559 (2005).

[56] P. J. Mohr, D. B. Newell, and B. N. Taylor, J. Phys. Chem. Ref. Data 45, 043102 (2016).

[57] F. Kochary, M. D. Aggarwal, A. K. Batra, R. Hawrami, D. Lianos, and A. Burger, J. Mater. Sci. Mater. Electron. 19, 1058 (2008).

[58] A. Abragam, The Principles of Nuclear Magnetism (Clarendon Press, Oxford, England, 1961).

[59] F. Bloch, Phys. Rev. 70, 460 (1946).

[60] V. V. Flambaum and J. S. M. Ginges, Phys. Rev. A 65, 032113 (2002).

[61] V. A. Dzuba, V. V. Flambaum, J. S. Ginges, and M. G. Kozlov, Phys. Rev. A 66, 012111 (2002).

[62] V. F. Dmitriev and R. A. Sen'kov, Phys. Rev. Lett. 91, 212303 (2003).

[63] V. V. Flambaum, D. DeMille, and M. G. Kozlov, Phys. Rev. Lett. 113, 103003 (2014).

[64] V. F. Dmitriev and R. A. Sen'kov, Phys. At. Nucl. 66, 1940 (2003).

[65] S. Ban, J. Dobaczewski, J. Engel, and A. Shukla, Phys. Rev. C 82, 015501 (2010).

[66] Y. V. Stadnik and V. V. Flambaum, Phys. Rev. D 89, 043522 (2014).

[67] I. Grinberg and A. M. Rappe, Phys. Rev. B 70, 220101(R) (2004).

[68] J. W. Foster, N. L. Rodd, and B. R. Safdi, Phys. Rev. D 97, 123006 (2018).
[69] B. Pelssers, M. Lawson, and N. L. Figueroa, The axion search simulation for lots of experiments (tassle), https:// github.com/thevorpalblade/tassle (2020).

[70] T. P. Robitaille et al. (Astropy Collaboration), Astron. Astrophys. 558, A33 (2013).

[71] A. M. Price-Whelan et al. (Astropy Collaboration), Astron. J. 156, 123 (2018).

[72] G. P. Centers, J. W. Blanchard, J. Conrad, N. L. Figueroa, A. Garcon, A. V. Gramolin, D. F. J. Kimball, M. Lawson, B. Pelssers, J. A. Smiga et al., arXiv:1905.13650.

[73] J. A. Ludlow and O. P. Sushkov, J. Phys. B 46, 085001 (2013).

[74] L. V. Skripnikov and A. V. Titov, J. Chem. Phys. 145, 054115 (2016).

[75] V. Andreev, D. G. Ang, D. DeMille, J. M. Doyle, G. Gabrielse, J. Haefner, N. R. Hutzler, Z. Lasner, C. Meisenhelder, B. R. O'Leary, C. D. Panda, A. D. West, E. P. West, and X. Wu, Nature (London) 562, 355 (2018).

[76] L.-S. Bouchard, A. O. Sushkov, D. Budker, J. J. Ford, and A. S. Lipton, Phys. Rev. A 77, 022102 (2008).

[77] L. Schiff, Phys. Rev. 132, 2194 (1963).

[78] P. G. Sandars, Phys. Rev. Lett. 19, 1396 (1967).

[79] O. P. Sushkov, V. V. Flambaum, and I. B. Khriplovich, Sov. Phys. JETP 87, 1521 (1984).

[80] V. V. Flambaum, I. B. Khriplovich, and O. P. Sushkov, Nucl. Phys. A449, 750 (1986).

[81] I. B. Khriplovich and S. K. Lamoreaux, CP Violation without Strangeness (Springer, Berlin, Heidelberg, 1997).

[82] V. V. Flambaum and V. A. Dzuba, Phys. Rev. A 101, 042504 (2020).

[83] K. Yanase and N. Shimizu, Phys. Rev. C 102, 065502 (2020).

[84] B. M. Brubaker, L. Zhong, S. K. Lamoreaux, K. W. Lehnert, and K. A. van Bibber, Phys. Rev. D 96, 123008 (2017).

[85] T. G. Castner, Phys. Rev. 115, 1506 (1959).

[86] W. DeRocco, P. W. Graham, and S. Rajendran, Phys. Rev. D 102, 075015 (2020).

[87] N. Bar, K. Blum, and G. D’Amico, Phys. Rev. D 101, 123025 (2020).

[88] G. Vasilakis, J. M. Brown, T. W. Kornack, and M. V. Romalis, Phys. Rev. Lett. 103, 261801 (2009).

[89] E. G. Adelberger and W. A. Terrano, Phys. Rev. Lett. 123, 169001 (2019).

[90] T. Wu, J. W. Blanchard, G. P. Centers, N. L. Figueroa, A. Garcon, P. W. Graham, Derek F. Jackson Kimball, S. Rajendran, Y. V. Stadnik, A. O. Sushkov, A. Wickenbrock, and D. Budker, Phys. Rev. Lett. 123, 169002 (2019).

[91] W. A. Terrano, E. G. Adelberger, C. A. Hagedorn, and B. R. Heckel, Phys. Rev. Lett. 122, 231301 (2019).

[92] S. Chaudhuri, Ph. D. thesis, 2019. 